Supporting Information

\title{
An Implantable Depot that Can Generate Oxygen In Situ for Overcoming Hypoxia-Induced Resistance to Anticancer Drugs in Chemotherapy
}

Chieh-Cheng Huang, Wei-Tso Chia, Ming-Fan Chung, Kun-Ju Lin, Chun-Wen Hsiao, Chuan Jin, Woon-Hui Lim, Chun-Chieh Chen, and Hsing-Wen Sung* 


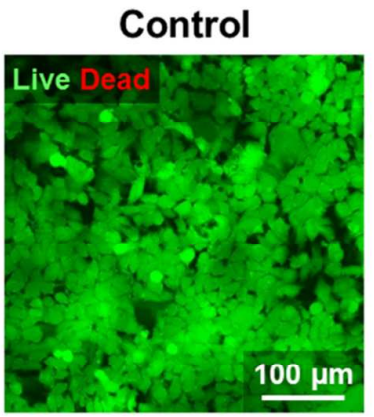

5 Pellets $/ m L$

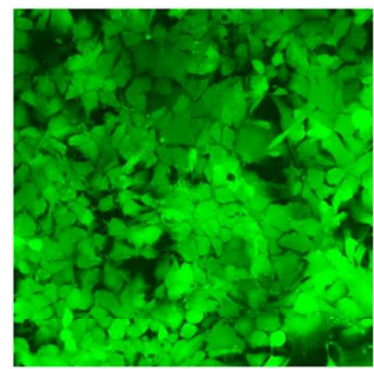

\section{Pellets $/ \mathrm{mL}$}

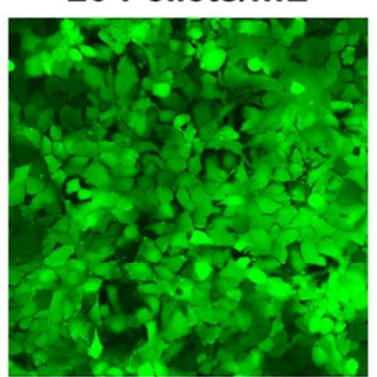

30 Pellets $/ \mathrm{mL}$

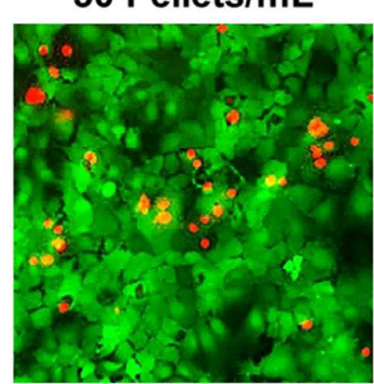

10 Pellets $/ m L$
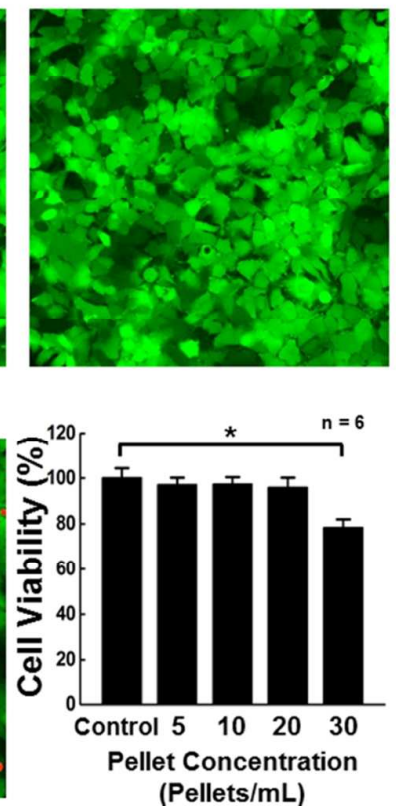

Figure S1. CLSM images of live/dead staining and results of MTT assay of Hep3B cells that were treated with various concentrations of test alginate pellets. ${ }^{*} P<0.05$. 


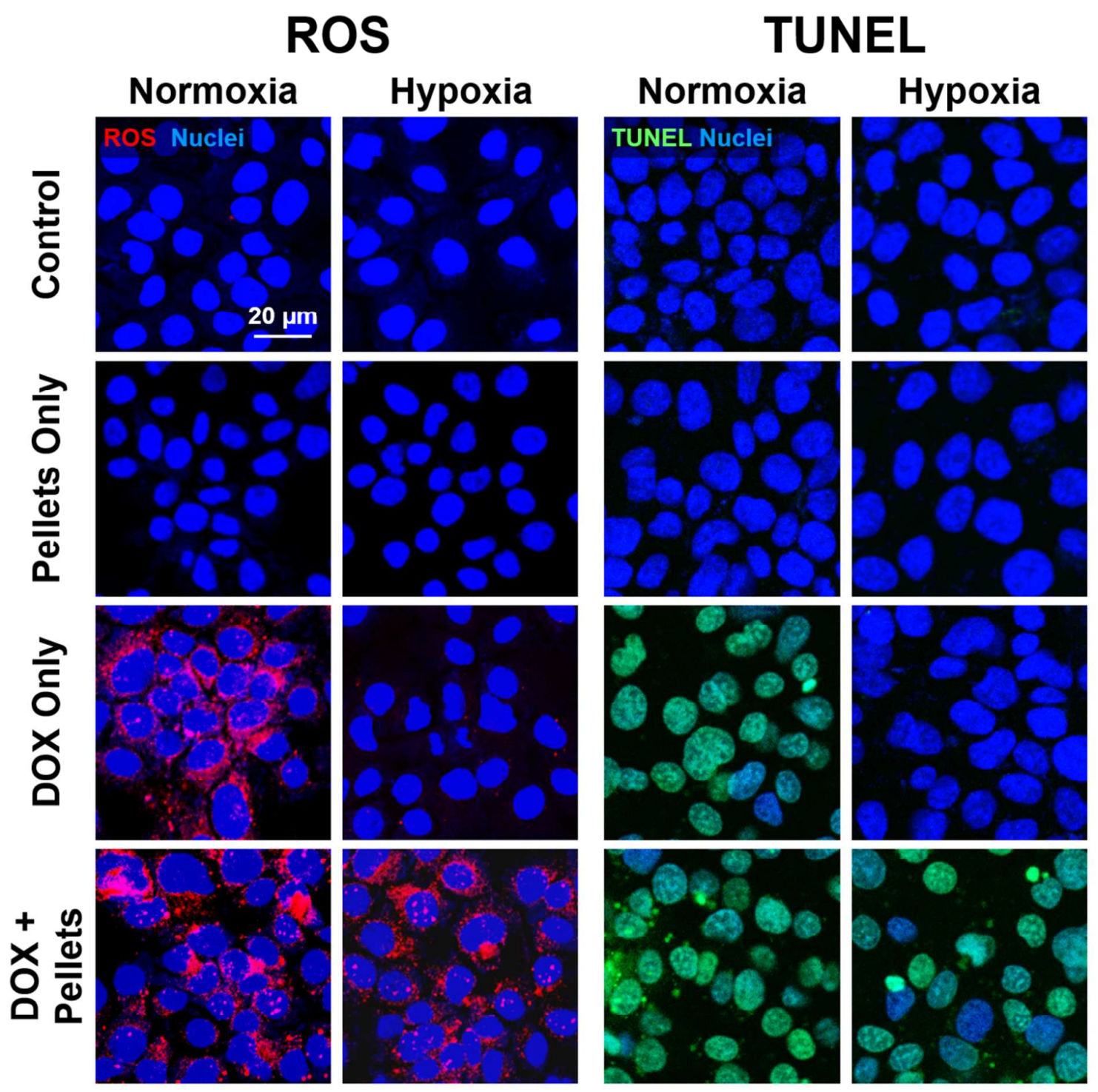

Figure S2. CLSM fluorescence images of intracellular production of ROS in Hep3B cells and images of their TUNEL staining following various treatments.

\section{Experimental Procedures}

Materials: Alginate (MW 30-70 kDa), calcium peroxide $\left(\mathrm{CaO}_{2}\right)$, calcium chloride $\left(\mathrm{CaCl}_{2}\right)$, and catalase from bovine liver were purchased from Sigma-Aldrich (St. Louis, MO, USA), while doxorubicin (DOX) was obtained from Fisher Scientific (Waltham, MA, USA).

Hep3B cells were acquired from the Bioresource Collection and Research Center, Food Industry Research and Development Institute, Hsinchu, Taiwan. Cell culture reagents were 
purchased from Life Technologies (Carlsbad, CA, USA). All other used chemicals and reagents were of analytical grade.

Preparation and Characterization of Oxygen-Generating Depot: The oxygen-generating depot that was developed in this work was prepared by dropping a $6 \%$ alginate solution that contained $7.5 \mathrm{mg} / \mathrm{mL} \mathrm{CaO}_{2}$ and $20,000 \mathrm{U} / \mathrm{mL}$ catalase into a gently stirred $\mathrm{CaCl}_{2}$ bath $(0.18$ $\mathrm{mM}$ ) using a 25-gauge needle to form $\mathrm{Ca}^{2+}$-crosslinked microencapsulated pellets. The alginate pellets thus formed were allowed to crosslink for $10 \mathrm{~min}$, harvested by filtration, rinsed with deionized water, and air-dried for subsequent assays.

The as-prepared alginate pellets were characterized by immersing them in a culture medium (20 pellets $/ \mathrm{mL})$ that had been pretreated with $1 \% \mathrm{O}_{2}$ for $24 \mathrm{~h}$. Following exposure to the culture medium, the formation of oxygen in the test pellets was grossly examined and photographed under a microscope (Eclipse E800, Nikon, Tokyo, Japan). The decomposition of the $\mathrm{CaO}_{2}$ that was encapsulated in the pellets was studied by monitoring changes in the $\mathrm{X}$ ray diffraction patterns between predetermined times using a powder diffractometer (Rigaku $\mathrm{D} /$ max-RB, Tokyo, Japan). The concentrations of oxygen and $\mathrm{H}_{2} \mathrm{O}_{2}$ that were generated in the reaction were quantified using a dissolved oxygen meter (HI 2400, Hanna Instruments, Smithfield, RI, USA) and the Amplex Red (Life Technologies) assay, respectively. Additionally, variations in the environmental $\mathrm{pH}$ of the test medium were measured using a pH meter (S47-K, Mettler Toledo, Greifensee, Switzerland).

Cellular Uptake of DOX and Induced Cytotoxicity: The effect of hypoxia on the cellular uptake of DOX was investigated using Hep3B cells. In this study, cells were incubated with DOX $(10 \mu \mathrm{M})$ under normoxic $\left(21 \% \mathrm{O}_{2}\right)$ or hypoxic $\left(1 \% \mathrm{O}_{2}\right)$ conditions for various periods. Following incubation, the cells were washed with prewarmed phosphate-buffered saline, fixed in 4\% paraformaldehyde, and analyzed using a flow cytometer (Cell Lab Quanta SC, Beckman Coulter, Inc., Fullerton, CA, USA) to determine the intracellular accumulation of DOX. 
The viability of the test cells that had been treated with various concentrations of DOX for $24 \mathrm{~h}$ in the normoxic or hypoxic culture was evaluated using the MTT assay, in which the treated cells were incubated in a medium that contained $1 \mathrm{mg} / \mathrm{mL}$ MTT (3-(4,5dimethylthiazol-2-yl)-2,5-diphenyltetrazolium bromide; Sigma-Aldrich) at $37{ }^{\circ} \mathrm{C}$. Four hours later, the metabolized MTT was dissolved in dimethyl sulfoxide, and its absorbance at $570 \mathrm{~nm}$ was then measured using a multi-mode microplate reader (SpectraMax M5, Molecular Devices, Sunnyvale, CA, USA).

Cytotoxicity of Test Alginate Pellets: The cytotoxicity of the test alginate pellets was evaluated in vitro by incubating Hep3B cells with various concentrations thereof $(0,5,10,20$, and 30 pellets $/ \mathrm{mL}$ ). Twenty-four hours after incubation, cell viability was qualitatively examined using a live/dead staining method that was based on calcein-AM and ethidium homodimer (LIVE/DEAD Viability/Cytotoxicity Kit, Life Technologies) and quantified by the MTT assay. In live/dead staining, calcein-AM is enzymatically converted into green fluorescent calcein in live cells, while ethidium homodimer stains the nuclei of dead cells with red fluorescence. ${ }^{1}$ Following cell staining, test samples herein were observed under a confocal laser scanning microscope (CLSM; LSM 780, Carl Zeiss, Jena, Germany).

Alleviation of Cellular Hypoxia by Alginate Pellets: The alleviation of hypoxia in Hep3B cells that had been hypoxically cultured in $2 \mathrm{D}$ or $3 \mathrm{D}$ format by various doses $(0,5$, and 20 pellets $/ \mathrm{mL}$ ) of test alginate pellets was investigated. Following treatment, cells were stained with antibodies against hypoxia-inducible factor (HIF)-1 $\alpha$ (Abcam, Cambridge, MA, USA) or HypoxiSense 680 (PerkinElmer, Waltham, MA, USA) to evaluate their degree of hypoxia. The stained cells were examined by CLSM, and their fluorescence intensities were analyzed using ImageJ software (National Institutes of Health, Bethesda, MD, USA) and normalized to those of control cells that had been cultured under standard oxygen tension.

The ability of the alginate pellets to allay the hypoxia-induced resistance of tumor cells to DOX was also investigated. Briefly, following $24 \mathrm{~h}$ of hypoxic incubation, cells were exposed 
to $\operatorname{DOX}(10 \mu \mathrm{M})+$ alginate pellets $(20$ pellets $/ \mathrm{mL})$ under hypoxic culture conditions for $24 \mathrm{~h}$. The intracellular production of reactive oxygen species (ROS) was detected by treating the test cells with CellROX Deep Red Reagent (Life Technologies); upon oxidation, this reagent yields a red cytoplasmic fluorescence. ${ }^{2}$ The treated cells were fixed in $4 \%$ paraformaldehyde and then observed under a CLSM. To determine the apoptotic activity in cultured cells, TUNEL (terminal deoxynucleotidyl transferase dUTP nick end labeling) assay was conducted according to the manufacturer's instructions (Roche Diagnostics GmbH, Mannheim, Germany). The viability of the cells was further determined using the MTT assay.

Animal Study: All animal experiments in this investigation were carried out according to the "Guide for the Care and Use of Laboratory Animals" of the Institute of Laboratory Animal Resources, National Research Council, published by the National Academy Press in 1996. The Institutional Animal Care and Use Committee of National Tsing Hua University (Hsinchu, Taiwan) reviewed and approved the study protocols.

Nude mice (BALB/cAnN.Cg-Foxn1nu/CrlNarl, 6-8 weeks old) were purchased from the National Laboratory Animal Center, Taiwan. To produce the animal model, Hep3B cells $(5 \times$ $10^{6}$ cells in $100 \mu \mathrm{L}$ Matrigel; BD Biosciences, San Jose, CA, USA) were implanted subcutaneously in the right flank region of each nude mouse. The tumor volume was estimated as length $\times$ width $\times$ height $\times \pi / 6$, and these dimensions were measured using a pair of caliper. ${ }^{3}$ When the tumors had reached an average volume of $150-200 \mathrm{~mm}^{3}$, a small incision was made in the skin to expose them, and various concentrations $(0,10,20$, or 30 pellets $/ \mathrm{cm}^{3}$ ) of the test alginate pellets were implanted subcutaneously close to the tumors (n $=6$ per group). Twenty-four hours later, the mice were intravenously administered HypoxiSense 680 to detect the tumor hypoxia. After another $24 \mathrm{~h}$, the treated mice were sacrificed, and their tumors were retrieved and imaged using an in vivo imaging system (IVIS; Xenogen, Alameda, CA, USA). 
Animals whose tumors satisfied the size criterion were treated under one of the following test conditions ( $\mathrm{n}=6$ for each group); saline (untreated control), intravenous administration of DOX ( $2 \mathrm{mg}$ per $\mathrm{kg}$ body weight), subcutaneous implantation of pellets $\left(20\right.$ pellets $\left./ \mathrm{cm}^{3}\right)$, and implantation of pellets $\left(20\right.$ pellets $\left./ \mathrm{cm}^{3}\right)$ that was followed, $24 \mathrm{~h}$ later, by intravenous administration of DOX ( $2 \mathrm{mg}$ per $\mathrm{kg}$ body weight). This treatment process was repeated every seven days, until a total of three treatments had been performed. The tumor volumes and body weights in each test group were measured every other day and normalized to their initial values.

Toward the end of the repeated treatments, the mice were caused to fast overnight and then anesthetized using isoflurane $\left(2 \%\right.$ in $\left.100 \% \mathrm{O}_{2}\right)$, before $0.32 \mathrm{mCi}{ }^{18} \mathrm{~F}$-flourodeoxyglucose $\left({ }^{18} \mathrm{~F}-\mathrm{FDG}\right)$ in $100 \mu \mathrm{L}$ of saline was administered via their tail veins. A $10 \mathrm{~min}$ image acquisition was conducted one hour following ${ }^{18} \mathrm{~F}$-FDG injection using a positron emission tomography (PET) scanner (Inveon, Siemens Medical Solutions, Knoxville, TN, USA). Following the PET scan, X-ray computerized tomography (CT) images were obtained using NanoSPECT/CT (Bioscan, Washington, DC, USA). Finally, the mice were sacrificed, and the tumor tissues were retrieved, fixed in $10 \%$ phosphate-buffered formalin, embedded in paraffin, sectioned, and stained with hematoxylin-eosin or TUNEL.

Statistical analysis: All results are presented as mean \pm SD. The two-tailed Student $t$ test was utilized to compare the means of pairs of groups. One-way analysis of variation (ANOVA) was followed by the Bonferroni post hoc test to assess variations among three or more groups. Differences were regarded as statistically significant at $P<0.05$. 


\section{References}

(1) Huang, C. C.; Pan, W. Y.; Tseng, M. T.; Lin, K. J.; Yang, Y. P.; Tsai, H. W.; Hwang, S. M.; Chang, Y.; Wei, H. J.; Sung, H. W. Biomaterials 2016, 74, 53.

(2) Bender, A.; Desplats, P.; Spencer, B.; Rockenstein, E.; Adame, A.; Elstner, M.; Laub, C.; Mueller, S.; Koob, A. O.; Mante, M.; Pham, E.; Klopstock, T.; Masliah, E. PLoS ONE 2013, 8, e62277.

(3) Ahn, R. W.; Chen, F.; Chen, H.; Stern, S. T.; Clogston, J. D.; Patri, A. K.; Raja, M. R.; Swindell, E. P.; Parimi, V.; Cryns V. L., Clin. Cancer Res. 2010, 16, 3607. 